\title{
Phenotype-loci associations in networks of patients with rare disorders: application to assist in the diagnosis of novel clinical cases
}

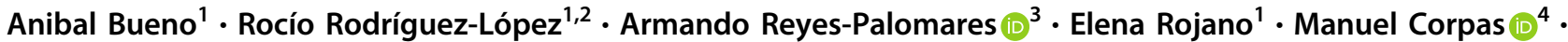 \\ Julián Nevado ${ }^{2,5} \cdot$ Pablo Lapunzina $^{2,5} \cdot$ Francisca Sánchez-Jiménez ${ }^{1,2} \cdot$ Juan A. G. Ranea ${ }^{1,2}$
}

Received: 21 June 2017 / Revised: 6 February 2018 / Accepted: 6 March 2018 / Published online: 26 June 2018

(c) The Author(s) 2018. This article is published with open access

\begin{abstract}
Copy number variations (CNVs) are genomic structural variations (deletions, duplications, or translocations) that represent the 4.8-9.5\% of human genome variation in healthy individuals. In some cases, CNVs can also lead to disease, being the etiology of many known rare genetic/genomic disorders. Despite the last advances in genomic sequencing and diagnosis, the pathological effects of many rare genetic variations remain unresolved, largely due to the low number of patients available for these cases, making it difficult to identify consistent patterns of genotype-phenotype relationships. We aimed to improve the identification of statistically consistent genotype-phenotype relationships by integrating all the genetic and clinical data of thousands of patients with rare genomic disorders (obtained from the DECIPHER database) into a phenotype-patient-genotype tripartite network. Then we assessed how our network approach could help in the characterization and diagnosis of novel cases in clinical genetics. The systematic approach implemented in this work is able to better define the relationships between phenotypes and specific loci, by exploiting large-scale association networks of phenotypes and genotypes in thousands of rare disease patients. The application of the described methodology facilitated the diagnosis of novel clinical cases, ranking phenotypes by locus specificity and reporting putative new clinical features that may suggest additional clinical follow-ups. In this work, the proof of concept developed over a set of novel clinical cases demonstrates that this network-based methodology might help improve the precision of patient clinical records and the characterization of rare syndromes.
\end{abstract}

Electronic supplementary material The online version of this article (https://doi.org/10.1038/s41431-018-0139-x) contains supplementary material, which is available to authorized users.

Juan A. G. Ranea

ranea@uma.es

1 Department of Molecular Biology and Biochemistry, University of Malaga, 29071 Malaga, Spain

2 CIBER de Enfermedades Raras, ISCIII, Madrid, Spain

3 European Molecular Biology Laboratory (EMBL) Heidelberg, Meyerhorfstrasse, 1, 69117 Heidelberg, Germany

4 Future Business Centre, King's Hedges Road, Cambridge CB4 2HY, UK

5 Instituto de Genética Médica y Molecular (INGEMM), IdiPAZ, Hospital Universitario La Paz, Universidad Autónoma de Madrid, Madrid, Spain

\section{Introduction}

Decades of advances in genomic technologies are increasing the accuracy in the field of genetic diagnosis. It is now widely accepted that deep phenotyping [1] and genotypic characterization of patients accelerates the identification of new genetic diseases and/or different disease subtypes with prognostic or therapeutic implications, as well as improves our understanding of human genetic diseases [2-4]. However, the accurate diagnosis of many genetic disorders becomes more complicated when patients show complex phenotypic profiles [5], when several genomic syndromes share clinical features among them, or when rare genetic aberrations affect an extremely low number of patients, as in rare diseases. Hence, key challenges for clinicians include the interpretation or classification of novel/extremely rare variants and the understanding of the phenotypic consequences of these genetic variations. A "genotype first" approach, in which patients are classified by a similar 
genomic rearrangement before a common clinical presentation is observed, has proven to be successful in characterizing the growing list of microdeletion/microduplication syndromes [6, 7].

The array-comparative genomic hybridization (aCGH) and single-nucleotide polymorphisms arrays (SNParrays), along with next-generation sequencing (NGS), are now the primary approaches used for copy number variation (CNV) detection [8]. CNVs are genomic structural variations that range from small variants $(1 \mathrm{~Kb})$ to larger structural changes (millions of nucleotides). These variations may correspond to deletions, duplications, or translocations found in genetic regions of individuals either inherited or by spontaneous occurrence (de novo), leading in some cases to disease [9]. CNVs are also present in healthy individuals, representing around the $4.8-9.5 \%$ of human genome variation in healthy individuals [8] of natural variation between genomes in the population. However, novel or inherited CNVs may be the cause of many disorders (as schizophrenia, Cron's disease, or autism) and their identification and analysis are used for the diagnosis and characterization of many chromosomal syndromes [10-12]. In some laboratories, microarrays are a legacy technology that will be replaced by NGS. However, the still growing number of patients genotyped with aCGH/ SNParrays platforms suggests a widespread usage of this technology. Indeed, public databases such as DECIPHER $[4,9,13]$ show a significant amount of data originating from aCGH and SNParray technologies in recent years.

Nowadays, the complete identification of the phenotypic consequences of a given CNV remains challenging. Thus, it is imperative that new significant advances are achieved in the characterization of the genetic regions and molecular mechanisms controlling phenotypic expression.

To help with the characterization of molecular relationships between different phenotypes and microvariants, we aimed to apply principles of network medicine [14-18] to find the consequences of variants and their association with diseases. To this end, we focused on the development of a computational approach via tripartite networks made of three types of nodes: variants (CNVs), patients, and phenotypes. We used the DECIPHER database, a global repository of clinical patient data, as a resource for a systematic analysis and characterization of CNVs that are likely to affect function [4]. DECIPHER is a valuable resource that offers the phenotype and genotype records of a sizable number of patients with low prevalent genomic disorders, collected from more than 200 institutions from around the world [4, 9, 13]. Most patients with de novo CNVs in the DECIPHER database correspond to pediatric disorders related to developmental delay, mental retardation, or congenital structural anomalies [5, 19]. Along with CNVs, DECIPHER provides the pathological phenotypic profiles of the patients. This information is stored using a normalized vocabulary of phenotypes: the human phenotype ontology (HPO) [20], that facilitates the analysis and comparison between patient symptomatologies. In order to study the genotype-phenotype relationships in this dataset, we exploited the associations in our purposely built tripartite networks using the subset of patients presenting de novo CNVs in DECIPHER, identifying significant associations between mutated regions and pathological phenotypes. These phenotype-locus associations have been used to assess the potential of our network approach for assisting in the diagnosis of novel uncharacterized rare cases in clinical routine. This approach shows the potential of integrating information from thousands of characterized cases to identify novel genotype-phenotype patterns in rare and isolated cases with very scarce information to compare with.

\section{Materials and methods}

\section{Source of datasets for building the networks}

We used the de novo CNVs from DECIPHER patients with rare genomic disorders annotated and with available HPO terms (version 2014-05-08, mapped to the hg19 genome reference) through a Data Access Agreement with the database consortium. All phenotype and genotype data belong to patients that have provided informed consent to share their data in an anonymized way. These include the set of HPO terms annotated for each patient and their respective associated CNVs. The deletions subnetwork includes 2436 de novo CNVs from 2301 patients and 1795 HPO phenotypes. Duplications subnetwork is formed by 1114 de novo CNVs from 1013 patients, including 1160 HPO terms. DECIPHER selects the potential pathological CNVs, removing those observed in control populations. The DECIPHER 2014 dataset version was used to validate our hypothesis, because this version did not include data from the patients used here as novel clinical cases, as they were included in later versions (see next section), allowing us to test the feasibility of the network approach presented in this work. In our approach, we analyzed two types of relationships: (1) patients and genotypes (by CNVs) and (2) patients and phenotypes (by HPO terms). We subdivided CNVs into deletions and duplications, as we have previously observed that they may have different effects when affecting the same region (for example, in 19p13.3 and $19 \mathrm{p} 13.13$ microdeletion/microduplication syndromes $[6,21]$, microdeletions lead to macrocephaly while microduplications to microcephaly). In addition, we focused only on de novo variants, as they are the ones more likely to be associated with pathological phenotypes and the largest genomic rearrangements [22]. 


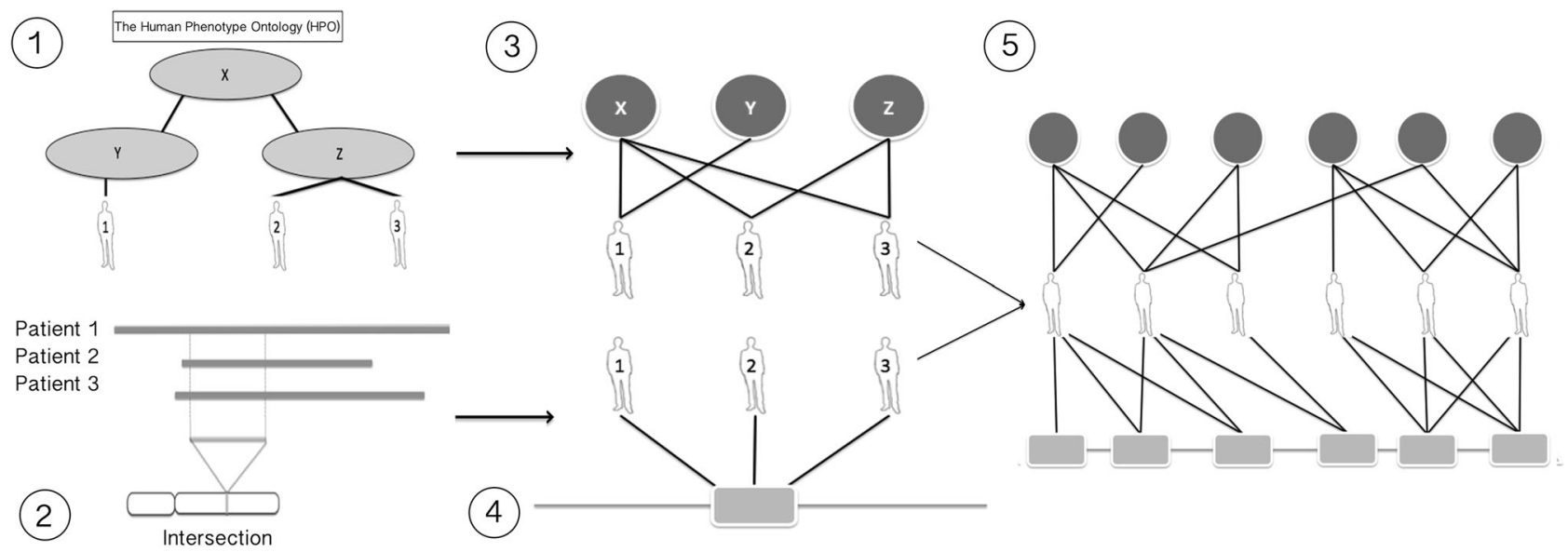

Fig. 1 Generation of a tripartite network using DECIPHER patient data. Circles represent phenotypes and rectangles loci. (1) Patients are phenotypically annotated using HPO terms; (2) a locus is defined as the chromosomal region where a set of patient CNVs overlap; (3) the HPOs-patients layers; (4) the patients-loci layers; (5) the final tripartite network

\section{Clinical case datasets used for testing our network approach}

In order to test the feasibility of our network-based approach (for assisting in clinical diagnosis of patients with gains and deletions), two cohorts of patients provided by the INGEMM (Institute of Medical and Molecular Genetics, Hospital La Paz, Madrid, Spain) were used. The data obtained were in the same format as DECIPHER data: an anonymised set of $\mathrm{CNVs}$ and their correspondingly annotated HPO terms per patient. Clinical investigations were performed according to the guidelines of the Declaration of Helsinki [23], and patient's data followed a strict ethical review process consisting of the signing of consent forms by patients (or their parents). We performed a proof of concept experiment of our technology in order to test if the associations found in the DECIPHER networks would help in the identification of the phenotypes associated to new pathological CNV cases from the INGEMM's sets of patients: (1) Single clinical cases: The first set of cases corresponds to a cohort of 293 patients (unpublished data) showing 519 genetic aberrations (312 deletions, 155 duplications, and 52 complex rearrangements), which were identified using oligonucleotide array CGH or SNParrays within 2010-2014 at the INGEMM. These patients were mainly referred to our clinics due to: intellectual disability, congenital malformations, and autistic spectrum disorder. (2) A group of patients sharing phenotype and genotype, describing a new microdeletion/microduplication syndrome: The second group of cases used was based on a specific syndrome characterization study, carried out by Nevado et al. [6], including 13 unrelated patients (with a total of 15 genomic rearrangements, distributed into 13 deletions and 2 duplications). Eleven of these patients had deletions and the remaining two duplications. The aCGH analysis together with clinical records showed that these patients shared phenotypic and genotypic features representing a novel interstitial microdeletion/microduplication syndrome [6]. Common features consist of: abnormal head circumference (macrocephaly for the deletions and microcephaly for the duplications), intellectual disability, developmental delay, hypotonia, speech delay, and some dysmorphic features.

\section{Microarrays analyses}

Array-CGH was performed using a custom oligonucleotide array (KaryoArray ${ }^{\circ}$ v3.0, $8 \times 60 \mathrm{~K}$, Agilent-Based Technologies, Santa Clara, CA) [24]. Briefly, this array has an average density of one probe per $9 \mathrm{~Kb}$ in clinically relevant regions (microdeletion/microduplication syndromes, subtelomeric, and pericentromeric regions) and one probe per $175 \mathrm{~Kb}$ in other genomic regions. In some cases, a genome-wide scan of high density 850,000 tag SNParrays was conducted on probands, using the commercial design Illumina CytoSNP-850k BeadChip according to the manufacturer's specifications (Illumina, San Diego, CA).

\section{Generating the network model}

Patient's HPO terms and de novo CNVs associations data files were downloaded from the DECIPHER ftp server. As HPO is organized as a hierarchical tree (Fig. 1, panel 1), each patient was associated, in the network model, to his/ her specific HPO terms (children) and all the parental terms above them in the HPO tree (Fig. 1, panel 3). A locus was defined as a SOR (small overlapping region) between patient CNVs used to build the network (Fig. 1, panel 2). A 
patient-loci network model (Fig. 1, panel 4) was generated connecting specific patients to loci. Panel 5 (Fig. 1) shows the integration of HPO phenotypes (red), loci (green), and patients. Thus, the layers of HPO terms and CNVs were connected by a middle layer of patients, forming a tripartite network, which was split in two halves; one for deletions and one for duplications. The deletions and duplications subnetworks generated 45,361 unique HPO term-patient/30,038 loci-patient associations and 17,010 unique HPO term-patient/10,888 loci-patient associations, respectively.

\section{Phenotype-genotype association measure calculation}

We used the Hypergeometric Index (HyI) to measure the degree of association between HPO terms and loci through patient nodes in the tripartite networks (Fig. 2). The HyI yields the minus log-transformed probability of having an equal or greater level of interaction between a given phenotype-locus pair than the one expected by chance [25]. This index is frequently used to measure associations in different areas: e.g., microarray functional analysis, image pairwise comparison, data vectors, and spatial analysis in mass spectrometry [26-28]. We explained in detail the mathematical method, how it behaves and how to access the source code and instructions in Supplementary Material, Section 1. We also made a cross-validation of the method (Supplementary Material, Section 2), tested its possible dependencies (Supplementary Material, Section 3) and found (a) a negative relationship between HyI values and HPO frequency, (b) a positive correlation between HPO prevalence and the number of associated loci, and (c) a lack of correlation between HyI values and patients/CNVs per locus. And finally we show an example, analyzing a prevalent phenotype (Supplementary Material, Section 4). The significance of the association increases with the HyI value, since the lower the probability of the observed phenotypelocus association to be due to random the higher the HyI score value. This metric also dampens the effects of large CNVs overlapping with many different small CNVs, in two ways: (1) a widely spread phenotype usually leads to low HyI scores and (2) the phenotype will be highly rated only if it is shared by a big number of patients in the same locus

$$
\mathrm{Hyl}_{\mathrm{AB}}=-\log _{10} \sum_{i=|N(\mathrm{~A}) \cap N(\mathrm{~B})|}^{\min (|N(\mathrm{~A}), N(\mathrm{~B})|)} \frac{\left(\begin{array}{c}
|N(\mathrm{~A})| \\
i
\end{array}\right) \cdot\left(\begin{array}{c}
\mathrm{n}_{\mathrm{y}}-|N(\mathrm{~A})| \\
|N(\mathrm{~B})|-i
\end{array}\right)}{\left(\begin{array}{c}
\mathrm{n}_{\mathrm{y}} \\
|N(\mathrm{~B})|
\end{array}\right)}
$$

Fig. 2 Hypergeometric Index (HyI) equation. 'A' represents a phenotype node and 'B' a locus node within the tripartite network
(SOR). Both facts, related to specificity, are discussed in Supplementary Material. An example of how HyI works is shown in Fig. 3, with two putative scenarios. Scenario 1 in Fig. 3 shows a phenotype connected to a locus via three different patients, but this phenotype has four more connections to other patients with genomic disorders located in different loci (high prevalent HPO phenotype). The HyI association value obtained in this case is low $(\mathrm{HyI}=0.001$; $p$-value $=0.99$ ). In Scenario 2 , a phenotype is also connected to a locus by three patients, but in this case the amount of connections to other patients and loci for that phenotype is low; this latter case represents a more specific phenotype-locus association, and therefore the significance is higher $(\mathrm{HyI}=0.942 ; p$-value $=0.11)$. In order to establish a significance threshold, for this study, we considered HyI value $\geq 2.0$ as significant HPO phenotype-loci associations (a $p$-value $\leq 0.01$ due to random).

\section{Measuring the association index between HPO phenotypes and loci in the whole DECIPHER network}

To calculate the significance of associations between HPO phenotypes and loci, we applied the hypergeometric association index (HyI) to the de novo deletions and de novo duplications tripartite subnetworks (see section above). We calculated the HyI association score for 600,234 different HPO term-locus pairs using the deletions subnetwork, and 175,956 using the duplications subnetwork. Some examples of HPO term-locus associations are shown in Table 1 and the rest of them are included in the Supplementary Material that accompanies this work.
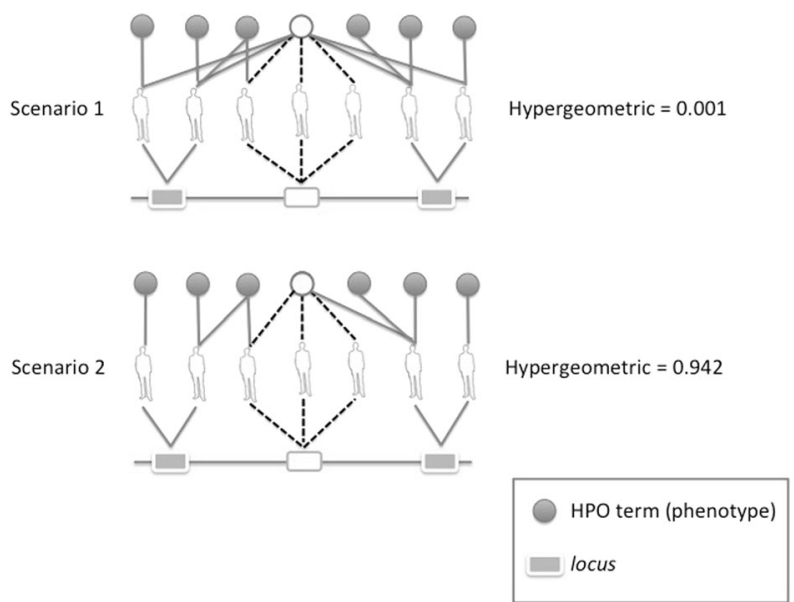

Fig. 3 Calculation of the Hypergeometric Index (HyI) in two scenarios within a tripartite network. Scenarios 1 and 2 show the HyI values for high and low prevalent phenotypes, respectively, connected to a locus via three patients 
Table 1 Examples of HPO-phenotypes vs. locus associations identified in the system

\begin{tabular}{llllll}
\hline HPO code & Phenotype & $\begin{array}{l}\text { Max HyI } \\
\text { (del.) }\end{array}$ & $\begin{array}{l}\text { Max HyI } \\
\text { (dup.) }\end{array}$ & Locus coordinates (del.) & Locus coordinates (dup.) \\
\hline HP:0002813 & $\begin{array}{l}\text { Abnormality of limb bone } \\
\text { morphology }\end{array}$ & 4.93 & 3.10 & Ch 7: 41613503-42807486 & Ch 9: 11818351-12709928 \\
HP:0000284 & Abnormality of ocular region & 3.39 & 1.65 & Ch 7: 41518389-41613502 & Ch Y: 945080-2654860 \\
HP:0000153 & Abnormality of the mouth & 3.50 & 1.94 & Ch 2: 200208169-200246437 & Ch 22: 40849826-41082043 \\
HP:0001315 & Reduced tendon reflexes & 3.16 & 3.54 & Ch 3: 6036656-6045520 & Ch 20: 29462074-29833608 \\
HP:0010477 & Aplasia of the bladder & - & 3.36 & - & Ch 17: 34817222-34817420 \\
HP:0001933 & Subcutaneous hemorrhage & 3.38 & - & Ch 21: 15398168-15412670 & - \\
HP:0200008 & Intestinal polyposis & 3.56 & - & Ch 10: 89717525-93614902 & - \\
HP:0001789 & Hydrops fetalis & 3.56 & - & Ch 13: 80378611-80386671 & - \\
HP:0003186 & Inverted nipples & 2.90 & 3.54 & Ch X: 455566-544731 & Ch 16: 75683739-78186860 \\
HP:0000699 & Diastema & 3.08 & 3.36 & Ch 5: 13750113-14064732 & Ch 7: 2290686-2996437 \\
HP:0010761 & Broad columella & 3.56 & - & Ch 19: 48066340-48270667 & - \\
HP:0008110 & Equinovarus deformity & 3.26 & 3.36 & Ch 16: 2038810-2124458 & Ch 16: 90148342-90148393 \\
HP:0002323 & Anencephaly & - & 3.36 & - & Ch 17: 34817222-34817420
\end{tabular}

Columns: (1) HPO code; (2) Phenotype description; (3) Maximun HyI obtained for the phenotype associated to a locus in the de novo deletions subnetwork; (4) Maximun HyI obtained for the phenotype associated to a locus in the de novo duplications subnetwork; (5) Chromosome id: the start and end coordinates (in bps in the hg19 genome reference) of the locus associated with the phenotype with the Max HyI value in the de novo deletions subnetwork; and (6) in the de novo duplications subnetwork

\section{Ranking putative phenotype/CNV associations in novel uncharacterized clinical cases}

We calculated HyI values for the de novo deletions and de novo duplications subnetworks. A dataset was set up to include all the HyI scores for all HPO phenotypes against all loci. We also used this dataset to identify phenotype-genotype associations, ranked by their $\mathrm{HyI}$ value, for new patients with CNVs that were not included in the DECIPHER dataset (Fig. 4).

\section{Other parameters used}

In addition to HyI association value, we also defined and calculated some additional parameters in order to estimate more accurately the significance of our results: Penetrance: We defined penetrance as the percentage of patients in the DECIPHER database with the same affected locus sharing the same HPO term. A penetrance of $100 \%$ means that all patients having affected the locus express the phenotype. Penetrance is a useful parameter for measuring the effects of combined genetic aberrations and the probability of showing the associated phenotype if a patient harbors a variant in that specific region. \% max: The ratio between the HyI value obtained for an HPO term associated to a locus and the maximum HyI value in the network for that phenotype. A \% $\max$ of $100 \%$ means that the HPO phenotype-locus HyI value is the maximum found for that HPO phenotype in the
Phenotype-Genotype specific association rank
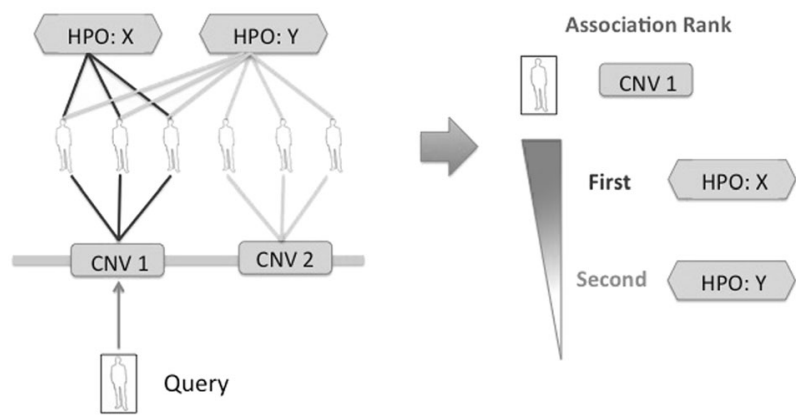

Fig. 4 Identification of phenotype-locus associations for new clinical cases. A CNV from a new patient (Query) is assigned to a locus (CNV 1) in the tripartite network by genomic overlap comparison (left side of the figure). All the phenotypes associated to patients are ranked based on their HyI association value to the query locus (right side)

whole genome. Locus overlap: The percentage of overlap (in base pairs) a CNV from a novel clinical case has with a locus present in our reference network. If this parameter is $100 \%$, that means that the "case" CNV is contained in the locus region. Locus overlap has to be taken into account in the interpretation of the data, since if the query patient just shared a tiny percentage with the studied locus, it may not affect the specific region responsible for a particular phenotype associated to that locus (even if the HyI value between the locus and the phenotype is high). 


\section{Results}

\section{Application of the networks analyses to novel clinical cases}

We used herein 293 clinical cases (patients), associated with 519 CNVs, diagnosed at the INGEMM (Institute of Medical and Molecular Genetics, Hospital La Paz, Madrid, Spain) during the period 2010-2014. For 258 out of the 293 clinical cases (88\%) our approach found at least an overlapping CNV with a pathological locus, and for each pathological locus a list of HPO phenotypes sorted by their HyI value was provided (as described in Methods, section "Ranking putative phenotype/CNV associations in novel uncharacterized clinical cases", Fig. 4). The resulting ranked list for each pathological locus only included HPO terms with a HyI value $\geq 2.0$ ( $p$-value $\leq 0.01)$. 17,096 significant $(\mathrm{HyI} \geq 2.0)$ associations were found involving 856 different phenotypes. A total of 381 out of the 1489 HPO terms (26\%) diagnosed by clinicians were also identified by our system associated to a patient's CNV in the de novo deletions subnetwork, and 252 out of $609(41 \%)$ in the de novo duplications subnetwork (Table 2). On the other hand, a total of 521 and 376 non-diagnosed HPO phenotypes, for deletion and duplication respectively, were identified by our method to be associated with disorders in the clinical cases (Table 2). These results indicate that this novel approach could be extensively used for differential diagnosis of novel clinical cases in order to find those phenotypes associated with single CNVs through comparison to the entire patient information integrated in the network generated from DECIPHER.

In order to illustrate the potential utility of this methodology for assisting in a genetic diagnosis, we select and discuss in detail three clinical cases (two deletions and one duplication) from our cohort (Table 3). Interestingly, patients presented as examples in Table 3 show a high level of similarity matching between phenotypes diagnosed by clinicians and those identified by our network association system. However, in the first case the system finds a couple of phenotypes: "Macrocephaly" and "Abnormality of joint mobility" (highlighted in dark in Table 3), which were not reported originally by the clinicians. Although, penetrance and \% max parameters are relatively low for both phenotypes, our results suggest the need for carrying out some additional clinical tests to confirm or discard these phenotypes in the patient.

The CNV of patient 2 has a region of about $100 \mathrm{~Kb}$ that does not match any locus (non-matching region coordinates -hg19-: $36410558-36510799$ bps in chr17). In this case, patient 2 was diagnosed with "Fetal choroid plexus cysts", an anomaly consisting of small fluid-filled structures within the choroid of the lateral ventricles of the fetal brain. These results suggest that the non-matching $\mathrm{CNV}$ region from the clinical case could harbor the genetic cause of the "Fetal choroid plexus cysts" diagnosed in this patient. This shows a potential use for the network approach in discriminating the particular regions associated with different phenotypes.

Finally, we show an example of a patient with a duplication (patient 3 in Table 3) where the clinicians observed two phenotypes: "Abnormal facial shape" and "Global developmental delay". The second phenotype was not detected by our system, but for the first one we detected up to eight ontologically related phenotypes with a significant HyI association value. The most specific phenotypes were the following: "Depressed nasal bridge", "Deviated nasal septum", "Malar flattening", and "Brittle hair". Just to note that, although "Brittle hair" has a significant HyI (2.82) it shows low values for penetrance and node overlap, so this should be carefully considered. Taking into account this information, we can infer that the "Abnormal facial shape" observed in this patient may be related to nose malformations and the structure of the zygomatic arch.

\section{Application of the methodology to a set of patients who share a novel non-recurrent microdeletion/ microduplication syndrome}

The same approach, described in the section above for single clinical cases, was also applied to a set of 13 patients

Table 2 Statistics of the comparison between the clinical records of the 293 patients with rare CNV genomic disorders and the HPO phenotypesloci associations identified by the system

\# HPO phenotypes diagnosed by the INGEMM clinicians for all the patients 


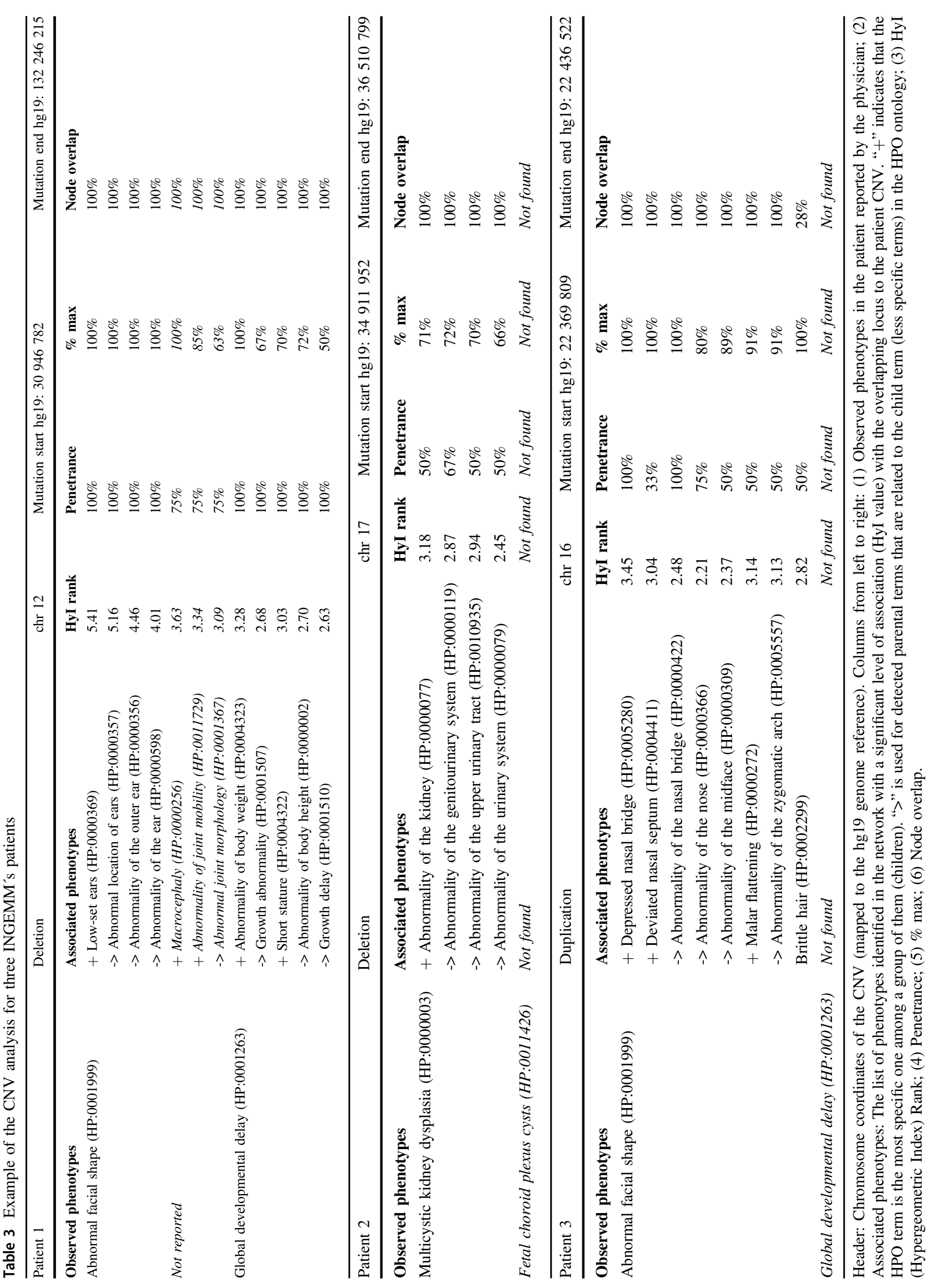


Table 4 Patients with the syndrome associated with CNVs in the $19 \mathrm{p} 13.3$ region

\begin{tabular}{|c|c|c|c|c|c|c|c|c|c|c|c|c|c|}
\hline \begin{tabular}{|l|} 
Patients \\
\end{tabular} & 1 & 2 & 3 & 4 & 5 & 6 & 7 & 8 & 9 & 10 & 11 & 12 & 13 \\
\hline \begin{tabular}{|l|l|} 
Type of CNV \\
To
\end{tabular} & & & & & & & & + & & + & & & \\
\hline \begin{tabular}{|l|} 
Gender \\
\end{tabular} & $\mathbf{F}$ & $F$ & $\mathrm{M}$ & $\mathbf{F}$ & 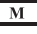 & $M$ & $F$ & $F$ & $F$ & $\mathbf{F}$ & M & $F$ & $F$ \\
\hline & & & & & & Growth & relopme & & & & & & \\
\hline \begin{tabular}{|l|} 
Psychom. \\
Develop. delay \\
(HPP:0002194, \\
HP:0011342, \\
H.001344)
\end{tabular} & $\mathrm{x}$ & $\mathrm{x}$ & $\mathrm{x}$ & $\mathrm{x}$ & $\mathrm{x}$ & $\mathrm{x}$ & $\mathrm{x}$ & $\mathrm{x}$ & $\mathrm{x}$ & $\mathrm{x}$ & $\mathrm{x}$ & $\mathrm{x}$ & $\mathrm{x}$ \\
\hline \begin{tabular}{|l} 
Intellectual \\
disability \\
(HP:0001256, \\
HP:0001249)
\end{tabular} & $\mathrm{x}$ & $\mathrm{x}$ & $\mathrm{x}$ & $\mathrm{x}$ & $\mathrm{x}$ & $\mathrm{x}$ & $\mathrm{x}$ & $\mathrm{x}$ & $\mathrm{x}$ & $\mathrm{x}$ & $\mathrm{x}$ & $\mathrm{x}$ & $\mathrm{x}$ \\
\hline \begin{tabular}{|l}
$\begin{array}{l}\text { Speech delay } \\
\text { (HP:0000750) }\end{array}$ \\
\end{tabular} & $\mathrm{x}$ & $\mathrm{x}$ & $\mathrm{x}$ & $\mathrm{x}$ & $\mathrm{x}$ & $\mathrm{x}$ & $\mathrm{x}$ & $\mathrm{x}$ & & $\mathrm{x}$ & & & $\mathrm{x}$ \\
\hline \begin{tabular}{|l|} 
Macro- \\
/Microcephaly \\
(HP:0000256, \\
HP:0000252)
\end{tabular} & $\mathrm{x}$ & $\mathrm{x}$ & $\mathrm{x}$ & $\mathrm{x}$ & $\mathrm{x}$ & & $\mathrm{x}$ & $\mathrm{x}$ & $\mathrm{x}$ & & $\mathrm{x}$ & $\mathrm{x}$ & $\mathrm{x}$ \\
\hline \begin{tabular}{|l|} 
Overgrowth \\
synd. testing \\
(HP:0001548) \\
\end{tabular} & & $\mathrm{x}$ & & & $\mathrm{x}$ & & & & & & & & $\mathrm{x}$ \\
\hline \begin{tabular}{|l|}
$\begin{array}{l}\text { Proportionate } \\
\text { short stature } \\
\text { (HP:0003508) }\end{array}$ \\
\end{tabular} & $\mathrm{x}$ & & $\mathrm{x}$ & $\mathrm{x}$ & & $\mathrm{x}$ & & $\mathrm{x}$ & & $\mathrm{x}$ & & & \\
\hline & & & & & & Abnori & the fac & & & & & & \\
\hline $\begin{array}{l}\text { Hypertelorism } \\
\text { (HP:0000316) }\end{array}$ & $\mathrm{x}$ & $\mathrm{x}$ & $\mathrm{x}$ & $\mathrm{x}$ & $\mathrm{x}$ & $\mathrm{x}$ & & & $\mathrm{x}$ & $\mathrm{x}$ & & & $\mathrm{x}$ \\
\hline \begin{tabular}{|l|}
$\begin{array}{l}\text { Downslanting } \\
\text { palpebral } \\
\text { fissures }\end{array}$ \\
\end{tabular} & & & $\mathrm{x}$ & & $\mathrm{x}$ & $\mathrm{x}$ & & & & $\mathrm{x}$ & & & \\
\hline \begin{tabular}{|l}
$\begin{array}{l}\text { Ptosis } \\
\text { (HP:0000508) }\end{array}$ \\
\end{tabular} & 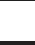 & & & & & $\mathrm{x}$ & & & & & & & \\
\hline \begin{tabular}{|l|}
$\begin{array}{l}\text { Epicanthal } \\
\text { folds } \\
(\mathrm{HP}: 0000286)\end{array}$ \\
\end{tabular} & $\mathrm{x}$ & $\mathrm{x}$ & & & & & & & & $\mathrm{x}$ & & & \\
\hline \begin{tabular}{|l|} 
Wide nasal \\
bridge \\
(HP:0000431)
\end{tabular} & $\mathrm{x}$ & $\mathrm{x}$ & $\mathrm{x}$ & $\mathrm{x}$ & $\mathrm{x}$ & $\mathrm{x}$ & $\mathrm{x}$ & $\mathrm{x}$ & & $\mathrm{x}$ & & & $\mathrm{x}$ \\
\hline \begin{tabular}{|l|} 
Depressed nose \\
and root \\
(HP:0005280)
\end{tabular} & $\mathrm{x}$ & $\mathrm{x}$ & $\mathrm{x}$ & & & $\mathrm{x}$ & $\mathrm{x}$ & & & $\mathrm{x}$ & & & $\mathrm{x}$ \\
\hline \begin{tabular}{|l|} 
Philtrum \\
anomalies \\
(HP:0000322)
\end{tabular} & $\mathrm{x}$ & $\mathrm{x}$ & & $\mathrm{x}$ & $\mathrm{x}$ & & & $\mathrm{x}$ & & & & & \\
\hline \begin{tabular}{|l|} 
Thin upper lip \\
$($ HP:0200086)
\end{tabular} & $\mathrm{x}$ & $\mathrm{x}$ & $\mathrm{x}$ & $\mathrm{x}$ & $\mathrm{x}$ & $\mathrm{x}$ & & $\mathrm{x}$ & & & & & \\
\hline \begin{tabular}{|l|} 
Ear anomalies \\
(HP:0000598)
\end{tabular} & $\mathrm{x}$ & $\mathrm{x}$ & $\mathrm{x}$ & $\mathrm{x}$ & $\mathrm{x}$ & $\mathrm{x}$ & & & & $\mathrm{x}$ & & & $\mathrm{x}$ \\
\hline \begin{tabular}{|l|}
$\begin{array}{l}\text { High or prom. } \\
\text { forehead } \\
\text { HPP:0011220) }\end{array}$ \\
\end{tabular} & $\mathrm{x}$ & $\mathrm{x}$ & $\mathrm{x}$ & $\mathrm{x}$ & $\mathrm{x}$ & $\mathrm{x}$ & $\mathrm{x}$ & $\mathrm{x}$ & $\mathrm{x}$ & $\mathrm{x}$ & & & $\mathrm{x}$ \\
\hline \begin{tabular}{|l|}
$\begin{array}{l}\text { Hypotonia } \\
\text { (HP:0001290) }\end{array}$ \\
\end{tabular} & $\mathrm{x}$ & $\mathrm{x}$ & $\mathrm{x}$ & $\mathrm{x}$ & $\mathrm{x}$ & $\mathrm{x}$ & $\mathrm{x}$ & $\mathrm{x}$ & & $\mathrm{x}$ & $\mathrm{x}$ & $\mathrm{x}$ & $\mathrm{x}$ \\
\hline \begin{tabular}{|l|} 
Behaviour \\
(HP:0000708, \\
HP:0000718)
\end{tabular} & $\mathrm{x}$ & & & & & $\mathrm{x}$ & & $\mathrm{x}$ & $\mathrm{x}$ & & $\mathrm{x}$ & & \\
\hline \begin{tabular}{|l|}
$\begin{array}{l}\text { Hearing } \\
\text { problems } \\
\text { (HP:0000365) }\end{array}$ \\
\end{tabular} & & & & & & & & & & & & & $\mathrm{x}$ \\
\hline & & & & & & Others & & & & & & & \\
\hline \begin{tabular}{|l|}
$\begin{array}{l}\text { Urinary reflux } \\
\text { (HP:00000076 }\end{array}$ \\
\end{tabular} & $\mathrm{x}$ & & $\mathrm{x}$ & & & & & & & & & & \\
\hline \begin{tabular}{|l|}
$\begin{array}{l}\text { Gastroesophag } \\
\text { eal reflux } \\
\text { (HP:0002020) }\end{array}$ \\
\end{tabular} & & & $\mathrm{x}$ & & $\mathrm{x}$ & & $\mathrm{x}$ & & & & & & $\mathrm{x}$ \\
\hline $\begin{array}{l}\text { Abnormal } \\
\text { fingers/toes } \\
\text { (HP:0006101, } \\
\text { HP:0001780, } \\
\text { HP:0001167) }\end{array}$ & $\mathrm{x}$ & & $\mathrm{x}$ & $\mathrm{x}$ & & & & $\mathrm{x}$ & & $\mathrm{x}$ & & & $\mathrm{x}$ \\
\hline \begin{tabular}{|l|} 
Feeding \\
problems \\
(HP:0008872)
\end{tabular} & $\mathrm{x}$ & & $\mathrm{x}$ & $\mathrm{x}$ & & & $\mathrm{x}$ & & & $\mathrm{x}$ & & & $\mathrm{x}$ \\
\hline \begin{tabular}{|l} 
Ophthalmologi \\
cabnormalities \\
(HP:0000504)
\end{tabular} & $\mathrm{x}$ & $\mathrm{x}$ & $\mathrm{x}$ & $\mathrm{x}$ & & & $\mathrm{x}$ & & & $\mathrm{x}$ & & & \\
\hline \begin{tabular}{|l|} 
Umbilical \\
hernias \\
(HP:0001537)
\end{tabular} & & & $\mathrm{x}$ & & $\mathrm{x}$ & & $\mathrm{x}$ & & & & & & $\mathrm{x}$ \\
\hline \begin{tabular}{|l|} 
Sleeping \\
disorders \\
(HP:0002360) \\
\end{tabular} & & & & & $\mathrm{x}$ & & $\mathrm{x}$ & & & & & & $\mathrm{x}$ \\
\hline \begin{tabular}{|l|} 
Heart disease \\
(HP:0001627) \\
\end{tabular} & & & $\mathrm{x}$ & & $\mathrm{x}$ & $\mathrm{x}$ & $\mathrm{x}$ & & $\mathrm{x}$ & $\mathrm{x}$ & & & $\mathrm{x}$ \\
\hline & & & & & & by thes & it not d & & & & & & \\
\hline $\begin{array}{l}\begin{array}{l}\text { Abnormality of } \\
\text { the kidney }\end{array} \\
\text { a }\end{array}$ & & & & & & & & & & & & & \\
\hline \begin{tabular}{|l|} 
Abnormality of \\
the penis
\end{tabular} & & & & & & & & & & & & & \\
\hline \begin{tabular}{|l}
$\begin{array}{l}\text { Abnormality of } \\
\text { connective } \\
\text { tissue }\end{array}$ \\
\end{tabular} & & & & & & & & & & & & & \\
\hline
\end{tabular}

The table shows the patient ids; Type of CNV: "_" for deletions and "+" for duplications. First column: phenotype descriptions and HPO codes. Boxes with $\mathrm{X}$ indicate that the phenotype has been previously diagnosed in the corresponding patient by the clinician examination. Phenotypes found by the systemic approach with significant HyI values (HyI value $\geq 2.0 ; p$-value $\leq 0.01$ ) are represented by dark gray boxes, and those detected with lower HyI values by light gray boxes. Phenotypes are grouped in four general categories: Growth and development, neurology, others, and an extra category for those found by the system but not previously diagnosed 
with 15 CNVs (13 deletions and 2 duplications), previously classified by reverse genetics in the INGEMM clinical unit into a new microdeletion/microduplication syndrome located at the 19p13.3 genomic region [6]. All these patients share a number of phenotypes related to a similar CNV rearrangement (deletions and duplications). The summary of the comparison between phenotypes established by Nevado et al. [6] and the results of our analysis are shown in Table 4. These results show that our systematic approach was able to identify 37 out of the 178 diagnosed phenotypepatient associations for this syndrome $(21 \%)$ with significant HyI values $(\mathrm{HyI} \geq 2.0$; $p$-value $\leq 0.01)$. Although we recommend the use of HyI values above 2.0 to obtain highly reliable results, our system also provides, as additional information, results with lower values, which must be taken with caution and be evaluated for each particular case, considering additional information in order to make any inference. In this sense, 91 out of the 178 diagnosed phenotype-patient associations for this syndrome (51\%) were also detected by our system but with $\mathrm{HyI}<2.0$. Some of these cases correspond to prevalent phenotypes, such as "psychomotor development delay" or "intellectual disability". We differentiate both types of results by using different colors in the grids of Table 4. There is a set of phenotypes diagnosed in most patients with this syndrome that were also recurrently found by our systematic approach, with significant HyI values. For example: "Wide nasal bridge" ( 9 associations found by the system out of 10 patients diagnosed), "Gastro-esophageal reflux" (4 out of 4 diagnosed), "Umbilical hernias" (4 out of 4), "Heart disease" ( 5 out of 7 ), and "Feeding problems" (5 out of 6 ). As we showed previously, our system also found phenotypes associated with these CNVs in $46 \%$ of the patients with this syndrome that have not been reported in the patients' clinical records, such as: "abnormality of the kidney", "abnormality of the penis", and "abnormality of connective tissue". In a retrospective review (carried out after the application of our method to check our predictions directly in patients) of 38 of these patients with 19p13.3 microdeletions, renal anomalies were found in $26.31 \%$ of them, anomalies of the sexual organs in $21.05 \%$, and there were no known cases of abnormality of the connective tissue. These results support the potential of the system to assist clinical diagnosis (see Section 5, Supplementary Material).

\section{Discussion}

We developed and showed herein a new approach to assess relationships between genotypes (using CNVs) and phenotypes (using HPO terms) in order to help in the diagnosis of rare genomic syndromes. We used the HPO [2], which provides a structured, comprehensive, and well-defined set of over 11,000 terms that characterize phenotypic abnormalities seen in human disease [20]. Many algorithms and computational tools use the HPO extensively. In fact, it is useful for clinical differential diagnostics, as well as for the prioritization of candidate disease-associated genes in exome sequencing studies [29]. As an example, the web application Phenomizer [30], which analyzes relationships between human phenotypic abnormalities and diseases, makes use of HPO database.

The systematic mathematical approach implemented in this work is able to establish a fine-tuning of the relationships between phenotypes and specific loci by exploiting large-scale association networks of phenotypes and genotypes in thousands of patients with rare disorders and complex pathologies. Clinicians can directly associate the variants of the patients and their phenotypes when they cooccur in the same locus, but they cannot easily differentiate the specificity degree and the association statistical significance of each phenotype associated to each particular locus, as the system implemented in this work does. Our results clearly support the use of this tool to identify potential loci involved in genetic diseases within a database such as DECIPHER, whose approximately half of its patients are currently not associated to genetic syndromic entities. The application of the described methodology in a set of novel clinical cases, used as proof of concept, has shown a high potential to facilitate the diagnosis of these novel unsolved clinical cases, ranking phenotypes by locus specificity and reporting putative new phenotypes. Indeed, these phenotypes may suggest additional clinical explorations that could help improve the precision of patient diagnosis and the characterization of new rare syndromes, as evidenced. The results obtained here indicate that the comparative analysis of new clinical cases with variantphenotype associations identified in the network of formerly diagnosed patients could have important applications in the design of customized arrays and NGS approaches for genetic-variant diagnosis, as well as in the search of candidate genes associated to the different mutated genomic regions observed in patients.

Currently available methodology for genetic diagnosis include PhenIX [31], Phenomantics [32], eXtasy [33], PHIVE, hiPHIVE [34, 35], Phevor [36], Phen-Gen [37], and OMIM-Explorer [29]. These tools provide phenotypically supported annotations to particular variants detected in patients, yielding rankings for pathological variants or genes. Some of these methods (PhenIX, PHIVE, hiPHIVE, or OMIM-Explorer) may provide, directly or indirectly, a guide for disease diagnosis. Particularly useful is Phenomizer, which compares diseases and patient phenotypes using the Online Mendelian Inheritance in Man (OMIM) disease catalog [30]. However, OMIM-Explorer is the only tool that provides phenotype suggestion for differential 
presumptive diagnosis, as the system presented in this work also does. The main difference between OMIM-Explorer and our network-approach is that the former uses available annotations of: genes, variants, diseases, and phenotypes in OMIM and other similar databases, while in this work the variants-phenotypes associations are directly inferred from a network of individual patients. In addition, another important difference in our tool is that it manages a tripartite (variants-patients-phenotypes) network made of pathological de novo CNVs present in patients with rare genomic disorders from the DECIPHER database. This characteristic makes the DECIPHER patients network approach presented here especially suitable for the comparative diagnosis of rare and orphan diseases with a genetic origin $(80 \%$ of all rare diseases), a clinical field that is specially challenging due to the low number of patients and the lack of computational methods for helping diagnosis [38-40].

Acknowledgements This work was supported by the European Commission, EU-FP7-Systems Microscopy Network of Excellence [258068], the Spanish Ministry of Economy and Competitiveness with European Regional Development Fund [SAF2016-78041-C2-1-R], and the Andalusian Government with European Regional Development Fund [CTS-486]. The CIBERER is an initiative from the Instituto de Salud Carlos III. REDES/FIBHULP08 from Hospital la PAZ, ENDOSCREEN (S2011/BMD-23969) from Comunidad Autonóma de Madrid, FIS 011/2491 from ISCIII. Thanks to FEDER grants and the EUCID-COST Action BM-1208. ARP is recipient of a postdoctoral fellowship granted by Fundación Ramón Areces. This study makes use of data generated by the DECIPHER Consortium. A full list of centres who contributed to the generation of the data is available from https://decipher.sanger.ac.uk/ and via email from decipher@sanger.ac. uk. Funding for the project was provided by the Wellcome Trust. DECIPHER Consortium bear no responsibility for the analysis of the data made in this work

\section{Compliance with ethical standards}

Conflict of interest The authors declare that they have no conflict of interest.

Open Access This article is licensed under a Creative Commons Attribution 4.0 International License, which permits use, sharing, adaptation, distribution and reproduction in any medium or format, as long as you give appropriate credit to the original author(s) and the source, provide a link to the Creative Commons license, and indicate if changes were made. The images or other third party material in this article are included in the article's Creative Commons license, unless indicated otherwise in a credit line to the material. If material is not included in the article's Creative Commons license and your intended use is not permitted by statutory regulation or exceeds the permitted use, you will need to obtain permission directly from the copyright holder. To view a copy of this license, visit http://creativecommons. org/licenses/by/4.0/.

\section{References}

1. Robinson PN. Deep phenotyping for precision medicine. Hum Mutat. 2012;33:777-80.
2. Köhler S, Doelken SC, Mungall CJ, et al. The human phenotype ontology project: linking molecular biology and disease through phenotype data. Nucleic Acids Res. 2014;42:D966-74.

3. Köhler S, Vasilevsky NA, Engelstad M, et al. The human phenotype ontology in 2017. Nucleic Acids Res. 2017;45: D865-76.

4. Firth HV, Richards SM, Bevan AP, et al. DECIPHER: database of chromosomal imbalance and phenotype in humans using ensembl resources. Am J Hum Genet. 2009;84:524-33.

5. Shaw-Smith C. Microarray based comparative genomic hybridisation (array-CGH) detects submicroscopic chromosomal deletions and duplications in patients with learning disability/mental retardation and dysmorphic features. J Med Genet. 2004;41: 241-8.

6. Nevado J, Rosenfeld JA, Mena R, et al. PIAS4 is associated with macro/microcephaly in the novel interstitial $19 \mathrm{p} 13.3$ microdeletion/microduplication syndrome. Eur J Hum Genet. 2015;23: $1615-26$

7. Tenorio J, Valencia M, Martinez-Glez V, et al. A new overgrowth syndrome is due to mutations in RNF125. Hum Mutat. 2014;35:1436-41.

8. Zarrei M, Macdonald JR, Merico D, Scherer SW, Mg C. A copy number variation map of the human genome. Nat Publ Gr. 2015;16:172-83.

9. Swaminathan GJ, Bragin E, Chatzimichali EA, et al. Decipher: web-based, community resource for clinical interpretation of rare variants in developmental disorders. Hum Mol Genet. 2012;21: R37-44. https://doi.org/10.1093/hmg/dds362.

10. Roizen NJ, Patterson D. Down's syndrome. Lancet. 2003;361: 1281-9.

11. Ballif BC, Hornor SA, Jenkins E, et al. Discovery of a previously unrecognized microdeletion syndrome of $16 \mathrm{p} 11.2-\mathrm{p} 12.2$. Nat Genet. 2007;39:1071-3.

12. Shaw-Smith C, Pittman AM, Willatt L, et al. Microdeletion encompassing MAPT at chromosome $17 \mathrm{q} 21.3$ is associated with developmental delay and learning disability. Nat Genet. 2006; 38:1032-7.

13. Bragin E, Chatzimichali EA, Wright CF, et al. DECIPHER: database for the interpretation of phenotype-linked plausibly pathogenic sequence and copy-number variation. Nucleic Acids Res. 2014;42:D993-1000. https://doi.org/10.1093/nar/gkt937.

14. Barabási A-L, Gulbahce N, Loscalzo J. Network medicine: a network-based approach to human disease. Nat Rev Genet. 2011;12:56-68.

15. Albert R, Barabasi AL. Statistical mechanics of complex networks. Rev Mod Phys. 2002;74:47-97.

16. Zhu X, Gerstein M, Snyder M. Getting connected: analysis and principles of biological networks. Genes Dev. 2007;21:1010-24.

17. Reyes-Palomares A, Bueno A, Rodríguez-López R, et al. Systematic identification of phenotypically enriched loci using a patient network of genomic disorders. BMC Genomics. 2016; 17:232.

18. Rodríguez-López R, Reyes-Palomares A, Sánchez-Jiménez F, Medina MÁ. PhenUMA: a tool for integrating the biomedical relationships among genes and diseases. BMC Bioinformatics. 2014; $15: 375$.

19. Lu X-Y, Phung MT, Shaw CA, et al. Genomic imbalances in neonates with birth defects: high detection rates by using chromosomal microarray analysis. Pediatrics. 2008;122:1310-8.

20. Robinson PN, Mundlos S. The human phenotype ontology. Clin Genet. 2010;77:525-34.

21. Dolan M, Mendelsohn NJ, Pierpont ME, Schimmenti LA, Berry SA, Hirsch B. A novel microdeletion/microduplication syndrome of 19p13.13. Genet Med. 2010;12:503-11.

22. Veltman JA, Brunner HG. De novo mutations in human genetic disease. Nat Rev Genet. 2012;13:565-75. 
23. World Medical Association. World Medical Association Declaration of Helsinki: ethical principles for medical research involving human subjects. J Am Med Assoc. 2013;310:2191-4.1.

24. Vallespín E, Palomares Bralo M, Mori MÁ, et al. Customized high resolution $\mathrm{CGH}$-array for clinical diagnosis reveals additional genomic imbalances in previous well-defined pathological samples. Am J Med Genet A. 2013;161:1950-60.

25. Bass JIF, Diallo A, Nelson J, Soto JM, Myers CL, Walhout AJM. Using networks to measure similarity between genes: association index selection. Nat Methods. 2013;10:1169-76.

26. Curtis RK, Oresic M, Vidal-Puig A. Pathways to the analysis of microarray data. Trends Biotechnol. 2005;23:429-35.

27. Kaddi CD, Parry RM, Wang MD. Multivariate hypergeometric similarity measure. IEEE/ACM Trans Comput Biol Bioinform. 2013;10:1505-16.

28. Kaddi C, Parry RM, Wang MD. Hypergeometric similarity measure for spatial analysis in tissue imaging mass spectrometry. 2011 IEEE Int Conf Bioinform Biomed. 2011;2011:604-7.

29. James RA, Campbell IM, Chen ES, et al. A visual and curatorial approach to clinical variant prioritization and disease gene discovery in genome-wide diagnostics. Genome Med. 2016;8:13.

30. Köhler S, Schulz MH, Krawitz P, et al. Clinical diagnostics in human genetics with semantic similarity searches in ontologies. Am J Hum Genet. 2009;85:457-64.

31. Torices R, Muñoz-Pajares AJ. PHENIX: an R package to estimate a size-controlled phenotypic integration index. Appl Plant Sci. 2015;3:1400104.

32. Masino AJ, Dechene ET, Dulik MC, et al. Clinical phenotypebased gene prioritization: an initial study using semantic similarity and the human phenotype ontology. BMC Bioinformatics. 2014;15:248.

33. Sifrim A, Popovic D, Tranchevent L-C, et al. eXtasy: variant prioritization by genomic data fusion. Nat Methods. 2013;10: 1083-4.

34. Robinson PN, Köhler S, Oellrich A, et al. Improved exome prioritization of disease genes through cross-species phenotype comparison. Genome Res. 2014;24:340-8.

35. Haendel MA, Vasilevsky N, Brush M, et al. Disease insights through cross-species phenotype comparisons. Mamm Genome. 2015;26:548-55.

36. Singleton MV, Guthery SL, Voelkerding KV, et al. Phevor combines multiple biomedical ontologies for accurate identification of disease-causing alleles in single individuals and small nuclear families. Am J Hum Genet. 2014;94:599-610.

37. Javed A, Agrawal S, Ng PC. Phen-Gen: combining phenotype and genotype to analyze rare disorders. Nat Methods. 2014;11:935-7.

38. Rare diseases need global solutions: new international initiatives in rare disease omics research I RD-CONNECT. http://rd-connect. eu/publications/rare-diseases-need-global-solutions-new-interna tional-initiatives-in-rare-disease-omics-research/. Accessed 18 Jun 2017.

39. Boycott KM, Vanstone MR, Bulman DE, MacKenzie AE. Raredisease genetics in the era of next-generation sequencing: discovery to translation. Nat Rev Genet. 2013;14:681-91.

40. Rath A, Olry A, Dhombres F, Brandt MM, Urbero B, Ayme S. Representation of rare diseases in health information systems: the orphanet approach to serve a wide range of end users. Hum Mutat. 2012;33:803-8. 